\title{
Life Style-Related Diseases of the Digestive System: from Molecular Mechanisms to Therapeutic Strategies: Preface
}

\author{
Atsushi Nakajima ${ }^{1, *}$ and Koichiro Wada ${ }^{2}$ \\ ${ }^{1}$ Gastroenterology Division, Yokohama City University School of Medicine, \\ 3-9 Fuku-ura, Kanazawa-ku, Yokohama 236-0004, Japan \\ ${ }^{2}$ Department of Pharmacology, Graduate School of Dentistry, Osaka University, \\ 1-8 Yamadaoka, Suita, Osaka 565-0871, Japan
}

Received July 10, 2007; Accepted September 10, 2007

Keywords: life style-related disease, colorectal cancer, metabolic syndrome, endocrine disruptor, hepatitis C virus (HCV), nonalcoholic steatohepatitis (NASH), peroxisome proliferator-activated receptor gamma (PPAR $\gamma$ )

Diabetes, obesity, atherosclerosis, hypertension, hyperlipidemia, myocardial infarction, stroke, hepatitis, fatty liver, colorectal cancer, pulmonary cancer, for example, were defined as life style-related diseases by the Japanese Ministry of Health, Labor and Welfare in 1996. Individual life style factors such as food, exercise, smoking, drinking, and stress are closely related to the onset of diseases. Recently, the number of patients with life style-related diseases has been markedly increasing year by year. Therefore, drastic steps including prevention and drug therapy of the diseases must be taken to prevent further increases in the number of patients with these diseases and to bring relief to those who have already developed these diseases. This is particularly urgent because the percentage of life style-related diseases of the digestive system, including the liver, intestine, and pancreas, has remarkably increased.

The purposes of the reviews presented here are to introduce the recent advances in elucidating the molecular mechanisms for these diseases and discuss the application of this information to develop an appropriate drug therapy for life style-related diseases of the digestive system. The first article presented by Takahashi and colleagues (doi: 10.1254/jphs.FM0070022) provides clear evidence showing that the metabolic syndrome is an important risk factor for colorectal cancer. The interesting article clarifies the relationship between aberrant crypt foci, pre-cancerous stage, and visceral fat,

*Corresponding author. nakajima-tky@umin.ac.jp

Published online in J-STAGE: October 6, 2007

doi: 10.1254/jphs.FM0070019 body mass, and serum lipid and suggests to us the application of peroxisome proliferator-activated receptor gamma $(\operatorname{PPAR} \gamma)$ ligand to prevent the aberrant crypt foci formation in human subjects. In the second article (doi: 10.1254/jphs.FM0070034), Wada and colleagues show the possibility that endocrine disruptors stimulate lipid accumulation in target cells and organs related to metabolic syndrome. We are exposed to various endocrine disruptors derived from synthetic detergents, plastics, oils, and so on, and therefore our bodies accumulate endocrine disruptors in adipose tissue, liver, and pancreas. Thus, these endocrine disruptors in our environment may be one of the risk factors for metabolic syndrome.

Hepatitis $\mathrm{C}$ virus (HCV) infection and the following hepatitis and cirrhosis are major liver diseases, and the patients have increased on a world-wide scale. The third article presented by Saito and colleagues (doi: 10.1254 /jphs.FM0070040) shows a new and unique model of hepatitis $\mathrm{C}$ virion production. Their data suggest that it may be possible to develop vaccines and therapeutic drugs for hepatitis C. In addition, Ikeda summarizes the screening for anti-HCV reagents using his unique cell culture system (doi: 10.1254/jphs.FM0070050). He shows the utility of combination therapy of statin with interferon. This exciting article provides us with a new therapeutic strategy of drugs for hepatitis C.

Nonalcoholic steatohepatitis is now recognized as one of the most common causes of liver disease. However, the molecular mechanisms of the onset of nonalcoholic steatohepatitis (NASH) are unclear. Yoneda and colleagues, based on their DNA microarray analysis, 
demonstrate the target genes involved in the onset of NASH (doi: 10.1254/jphs.FM0070063). They also show the therapeutic utility of a $\operatorname{PPAR} \gamma$ ligand to attenuate liver dysfunction.

This forum minireview is based on the symposium on "Life style-related diseases of the digestive system: from molecular mechanisms to therapeutic strategies" at the 80th Annual Meeting of The Japanese Pharmacological Society held on March 14, 2007, Nagoya. All the authors hope that this forum minireview helps the readers to understand the basic molecular mechanisms for these diseases and the application of this knowledge to develop appropriate drug therapies for these diseases. 